dure. ${ }^{1}$ The appropriate interval between operations is hard to define; LeMaire, Carter, and Coselli ${ }^{1}$ have advocated a 6-week recovery period whereas Safi and colleagues ${ }^{4}$ recommend performing the second-stage repair 4 weeks after the first stage. In patients who have had elephant trunk repair of the arch, a secondstage surgical approach via a left thoracotomy for the replacement of the DTA is usually performed. On the other hand, several groups have reported using endografts to complete the second stage of the procedure. ${ }^{1,5} \mathrm{We}$ chose endoluminal repair of the DTA aneurysm because we believed that it reduced procedural time, blood loss, length of intensive care unit stay, and hospital stay and that it is associated with lower rates of morbidity and mortality if compared with conventional open surgical procedures.

In our patient, the presence of an aneurysmal dilatation of ARSA origin made management of this already complex pathologic condition even more complicated. Most patients with ARSA are asymptomatic, but aneurysms in this location require surgical treatment since they can cause serious complications: distal embolization, rupture, and compression of neighboring structures. ${ }^{2,6}$ Because of the rareness of the ARSA aneurysms, surgical strategies are still unclear. We have performed a bilateral subclaviancarotid bypass after ETT to prepare the patient for the last step of the procedure: stent grafting of the DTA. We have modified the conventional 2-step repair of thoracic complex aneurysms adding a third stage to suit the peculiar features of our patient. The 3-stage approach has been effective and we believe that it can be safely performed in patients with diffuse aneurysms of the thoracic aorta involving an ARSA. Long-term evaluation in larger numbers of patients is necessary to verify the efficacy of this technique.

\section{References}

1. LeMaire SA, Carter SA, Coselli JS. The elephant trunk technique for staged repair of complex aneurysms of the entire thoracic aorta. Ann Thorac Surg. 2006;81:1561-9.

2. Kamiya H, Knobloch K, Lotz J, Bog A, Lichtenberg A, Hagl C, et al. Surgical treatment of aberrant right subclavian artery (arteria lusoria) aneurysm using three different methods. Ann Thorac Surg. 2006;82:187-90.

3. Borst HG, Walterbush G, Schaps D. Extensive aortic replacement using "elephant trunk" prosthesis. Thorac Cardiovasc Surg. 1983;31:37-40.

4. Safi HJ, Miller CC 3rd, Estrera AL, Huynh TT, Porat EE, Allen BS, et al. Staged repair of extensive aortic aneurysms: long-term experience with the elephant trunk technique. Ann Surg. 2004;240:677-85.

5. Greenberg RK, Haddad F, Svensson L, O'Neill S, Walker E, Lyden $\mathrm{SP}$, et al. Hybrid approaches to thoracic aortic aneurysms: the role of endovascular elephant trunk completion. Circulation. 2005;112: 2619-26

6. Austin EH, Wolfe WG. Aneurysm of aberrant subclavian artery with a review of the literature. J Vasc Surg. 1985;2:571-7.

\title{
Systemic thrombolysis for prosthetic valve thrombosis in the immediate postoperative period of major abdominal surgery
}

\author{
Manuel Ruiz-Bailén, MD, PhD, ${ }^{\mathrm{a}, \mathrm{b}}$ Benjamín Narbona-Carvo, $\mathrm{MD}^{\mathrm{c}}$ José Ángel Ramos-Cuadra, MD, ${ }^{\mathrm{d}}$ \\ Luis Rucabado-Aguilar, MD, ${ }^{a}$ Carmen López-Caler, MD, ${ }^{\mathrm{e}}$ and Francisco Javier Gómez-Jiménez, MD, Jaén, Almería, and \\ Granada, Spain
}

$\mathrm{T}$ hrombosis of a prosthetic valve is usually a cause for emergency cardiac surgery, particularly in the presence of a major contraindication for systemic thrombolysis. Recent surgery is a major contraindication for the administration of thrombolysis. Our objective is to describe the

From the Intensive Care Unit, Critical Care and Emergencies Department,

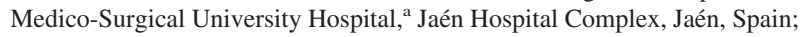
Departamento Ciencias de la Salud, Universidad de Jaén, ${ }^{b}$ Jaén, Spain; Surgery Department, Poniente Hospital, ${ }^{\mathrm{c}}$ El Ejido, Almería, Spain; Intensive Care Unit, Critical Care and Emergency Department, Torrecárdenas Hospital, ${ }^{\mathrm{d}}$ Almería, Spain; Intensive Care Unit, Critical Care and Emer-

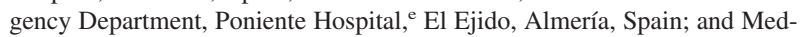
icine Department, University of Granada, ${ }^{\mathrm{f}}$ Granada, Spain.

Received for publication Sept 17, 2006; accepted for publication Oct 24, 2006.

Address for reprints: Dr. Manuel Ruiz-Bailén, C/. Las Torres 57, 23650 Torredonjimeno. Jaén, Spain (E-mail: mrb1604@teleline.es).

J Thorac Cardiovasc Surg 2007;133:801-3

$0022-5223 / \$ 32.00$

Copyright $\odot 2007$ by The American Association for Thoracic Surgery doi:10.1016/j.jtcvs.2006.10.037 administration of thrombolysis in a valve prosthesis after major surgery.

\section{Clinical Summary}

A 61-year-old male patient was admitted for lower intestinal obstruction. Sigmoidoscopy was performed and a tumor (moderately differentiated adenocarcinoma) of the upper rectum, $12 \mathrm{~cm}$ from the anal margin, was diagnosed. The patient had a metallic cardiac valve prosthesis (St Jude Medical, Inc, St Paul, Minn) in the mitral position and was being treated with acenocumarol. After reversal of the anticoagulation, the patient underwent surgery, a subtotal proctocolectomy with ileorectal anastomosis. The patient was admitted to the intensive care unit (ICU) because of hemodynamic instability in the first 24 hours, requiring high doses of dopamine and norepinephrine, with an initial diagnosis of distributive shock. Forty-eight hours after the operation, a transesophageal echocardiogram with hemodynamic assessment was performed, revealing prosthetic valve thrombosis with obstruction and abnormal disc motion of the medial hemidisc. A mean transmitral pressure gradient of $19.6 \mathrm{~mm} \mathrm{Hg}$ was observed (Figure 1). The Doppler echocardiographic hemodynamic study was compatible 


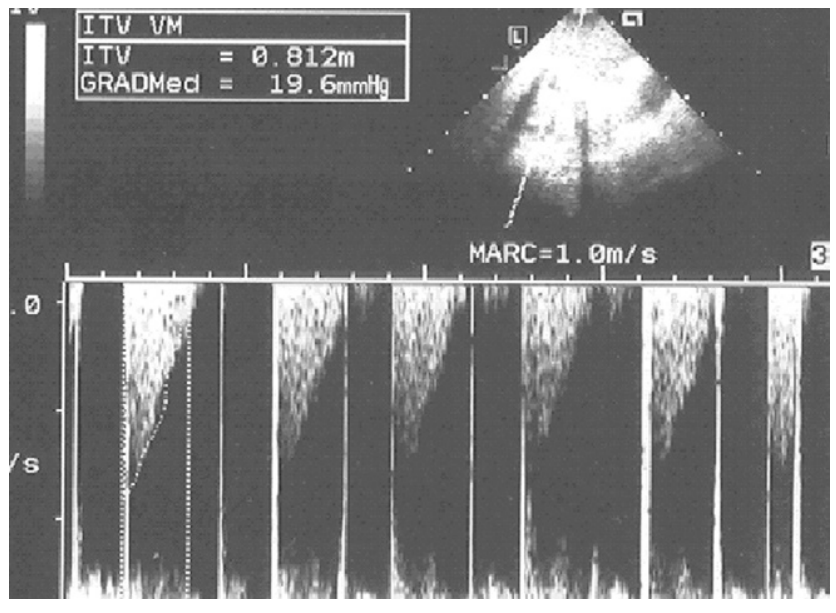

Figure 1. Mean transmitral gradient before the administration of thrombolysis. MARC, $1.0 \mathrm{~m} / \mathrm{s}$.

with severe cardiogenic shock, with a left atrial pressure of $27 \mathrm{~mm}$ $\mathrm{Hg}$ using the Kuecherer formula. ${ }^{1}$ The patient remained in hemodynamically unstable condition, with acute pulmonary edema and prerenal renal failure. Transfer to the reference hospital for surgery in the reference cardiac surgery department was rejected. Because of the persistence of cardiogenic shock, systemic thrombolysis (100 mg of alteplase over 120 minutes) was administered on the sixth postoperative day. A repeat transthoracic and transesophageal echocardiogram, performed at the end of the alteplase infusion, demonstrated disappearance of the prosthetic valve thrombosis and normalization of the transmitral gradient (Figure 2). The patient's hemodynamic situation normalized; extubation was possible 48 hours after administration of the alteplase, and the patient was discharged from the ICU. A later complication occurred in the form of an upper gastrointestinal hemorrhage resulting from an acute, self-limiting lesion of the gastric mucosa, which did not require blood transfusion. After transfer to the surgery ward, the remainder of the postoperative course was within normal limits. Follow-up at 6 months was normal.

\section{Discussion}

In the context of major abdominal surgery with a high risk of hemorrhage and the long-term administration of anticoagulant drugs, an option undertaken on occasion is the normalization of the prothrombin activity for the duration of surgery, while simultaneously administering prophylactic doses of enoxaparin. However, although the period the patient is without anticoagulation is relatively short, in patients with a high risk of thrombosis, as in the present case, in which the diagnosis was of adenocarcinoma, this period may be sufficient for a thrombotic event, such as prosthetic valve thrombosis, to develop. ${ }^{2}$

A further interesting problem in this discussion concerns reaching the correct diagnosis; the most frequent diagnosis, distributive shock resulting from cytokine release, was initially assumed. However, although the possibility of this situation existed, there was a clear cardiologic cause in this patient: prosthetic valve thrombosis. This case is a prime example of the benefit of echocardiography

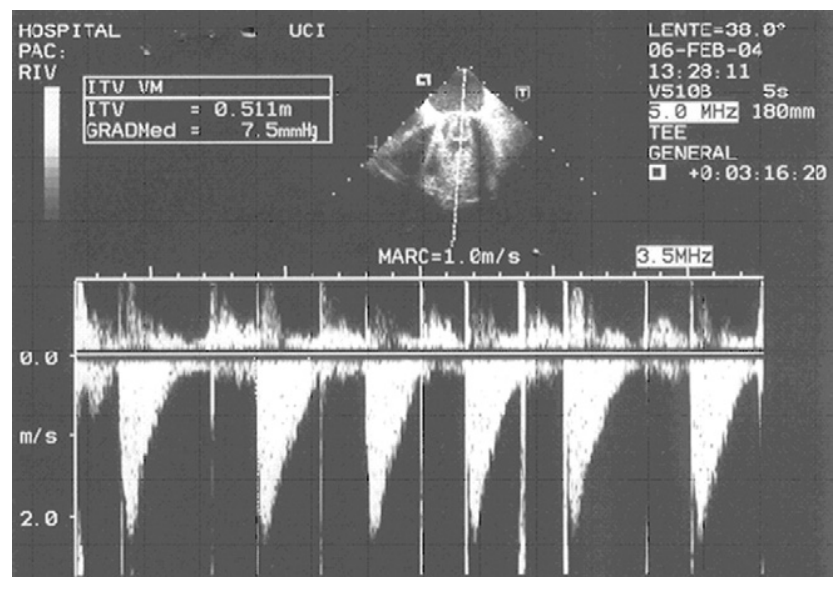

Figure 2. Mean transmitral gradient after the administration of thrombolysis. MARC, $1.0 \mathrm{~m} / \mathrm{s}$.

carried out in the ICU, being performed to assess the hemodynamic situation without suspecting the cardiologic cause a priori. This might encourage intensive care physicians to consider the need to use this technique routinely in hemodynamically unstable patients, as it is a diagnostic technique that has proven very effective and may change the therapeutic approach. ${ }^{3}$

Another problem consists in the therapeutic approach to be followed after the diagnosis of prosthetic valve thrombosis has been made in a patient in cardiogenic shock with multiorgan dysfunction. Thrombolysis may have a hemorrhagic effect and increase the emboligenic risk; however, it has been shown to be effective and efficient in the treatment of prosthetic valve thrombosis and has been proposed as the initial treatment. ${ }^{4,5}$

In patients with a high hemorrhagic risk (an absolute contraindication to systemic thrombolysis), as in the present case, the most straightforward option would be surgery. Our patient also was not a candidate for surgery, however, owing to an unacceptable surgical risk. In this case, and in similar cases, systemic thrombolysis may be administered as a final, desperate measure. Despite the absolute contraindication for thrombolysis in the immediate postoperative period, it has been administered at this time, and even during surgery itself, in many cases, in case series of surgical patients with pulmonary thromboembolism, and even in high-risk situations such as pregnancy. ${ }^{6}$ For this reason, despite an absolute contraindication for systemic thrombolysis, in specific cases such as this one, the risk of its use should be evaluated and compared with the natural course of the pathologic condition without its use.

\section{References}

1. Kuecherer HF, Muhiudeen IA, Kusumoto FM, Lee E, Moulinier LE, Cahalan MK, et al. Estimation of mean left atrial pressure from transesophageal pulsed Doppler echocardiography of pulmonary venous flow. Circulation. 1990;82:1127-39.

2. Ferreira I, Dos L, Tornos P, Nicolau I, Permanyer-Miralda G, SolerSoler J. Experience with enoxaparin in patients with mechanical heart valves who must withhold acenocumarol. Heart. 2003;89:527-30.

3. Colreavy FB, Donovan K, Lee KY, Weekes J. Transesophageal echocardiography in critically ill patients. Crit Care Med. 2002;30:989-96. 
4. Azpitarte J, Sanchez-Ramos J, Urda T, Vivancos R, Oyonarte JM, Malpartida F. Prosthetic valve thrombosis: which is the most appropriate initial therapy? Rev Esp Cardiol. 2001;54:1367-76.

5. Martin Herrero F, Sanchez Fernandez PL, Piedra Bustamante I, Morinigo Munoz JL, Nieto Ballestero F, Martin Luengo C. Mitral prosthesis thrombosis treated by fibrinolysis with accelerated administration of r-TPA. Rev Esp Cardiol. 2001;54:1448-51.

6. Ruiz Bailén M, Aguayo de Hoyos E, Ramos Cuadra JA. Thrombolysis during cardiopulmonary resuscitation in fulminant pulmonary embolism. A review. Crit Care Med. 2001;29:2211-9.

\title{
Left atrial epithelioid hemangioendothelioma
}

\author{
Milan Lisy, MD, ${ }^{a}$ Wolfram Beierlein, MD, ${ }^{a}$ Hartmut Müller, MD, PhD, ${ }^{b}$ Burkhard Bültmann, MD, $\mathrm{PhD},{ }^{\mathrm{b}}$ \\ and Gerhard Ziemer, MD, PhD, ${ }^{a}$ Tubingen, Germany
}

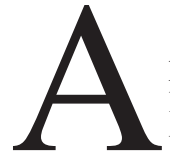

case of cardiac epithelioid hemangioendothelioma is presented, occurring as a clinically silent pediculated left atrial tumor in a 61-year-old male patient who underwent pancreaticoduodenectomy for carcinoma of the pancreas 3 years previously. The tumor was completely resected with the presumptive clinical diagnosis of atrial myxoma. Histologic and immunohistochemical investigations demonstrated a mesenchymal tumor with antigen positivity for hemangioendotheliomas. Epithelioid hemangioendotheliomas are rare vascular tumors of the heart, histologically characterized by capillary-sized vessels lined by proliferating plump, often multilayered, epitheliallike endothelial cells with cytoplasmic vacuoles. ${ }^{1}$ Similar to most other primary intracardiac tumors, epithelioid hemangioendotheliomas may be found incidentally or after causing congestive heart failure, pericardial effusions, outflow tract obstruction, or thromboembolic events. Because of the biologic behavior of epithelioid hemangioendotheliomas, they are regarded as fully malignant, rather than borderline neoplasms, and are to be resected radically to prevent metastatic embolic disease.

Primary cardiac tumors are rare and most commonly histologically benign, with only $30 \%$ of them exhibiting histologic signs of malignancy. ${ }^{2}$ Clinically, they may be found incidentally by chest radiogram or by causing dysrhythmia, congestive heart failure, pericardial effusions, outflow tract obstruction, or thromboembolic events. $^{3}$

The epithelioid hemangioendothelioma is histologically characterized by capillary-sized vessels lined by proliferating plump epithelial-like endothelial cells with cytoplasmic vacuoles, often multilayered. It is regarded as a malignant, rather than a borderline, neoplasm.

From the Department of Thoracic, Cardiac and Vascular Surgery, ${ }^{a}$ and Department of Pathology, ${ }^{\mathrm{b}}$ Tübingen University Hospital, Tubingen, Germany.

Received for publication Sept 11, 2006; accepted for publication Sept 28, 2006.

Address for reprints: Gerhard Ziemer, MD, PhD, Department of Thoracic, Cardiac and Vascular Surgery, Tübingen University Hospital, HoppeSeyler Straße 3, D-72076 Tübingen (E-mail: gdziemer@uni-tuebingen.de).

J Thorac Cardiovasc Surg 2007;133:803-4

$0022-5223 / \$ 32.00$

Copyright $\odot 2007$ by The American Association for Thoracic Surgery

doi:10.1016/j.jtcvs.2006.09.076

\section{Clinical Summary}

A 61-year-old man presented with nonspecific abdominal pain 3 years after he underwent a pancreaticoduodenectomy (Whipple's procedure) for adenocarcinoma of the pancreas. He had arterial hypertension, noninsulin-dependent diabetes mellitus, and sleepinduced apnea syndrome. His medication included metoprolol, captopril, allopurinol, and pancreatic enzyme supplements. Specific tumor markers for the pancreas were normal (carcinoembryonic antigen $3 \mu \mathrm{g} / \mathrm{L}$, cancer antigen 19-9 $5 \mathrm{kU} / \mathrm{L}$ ). His electrocardiogram showed sinus rhythm and first-degree atrioventricular block, without ST-T anomalies.

Computed tomography excluded intraabdominal masses and remote lymphatic metastases of his previous pancreatic carcinoma. The chest computed tomography scan showed a $3 \times 3-\mathrm{cm}$ noncalcified mass on the posterolateral wall of the left atrium, a rather atypical site for myxoma.

Transthoracic and transesophageal echocardiography demonstrated good left ventricle function without hypertrophy and a mobile pediculated left atrial mass displaying typical features of a myxoma. The mitral valve was not involved.

Angiocardiography showed neither valvular nor coronary abnormalities, demonstrating the vascular supply of the atrial tumor originating from the proximal circumflex coronary artery.

Elective surgery was performed through a midline sternotomy using hypothermic cardiopulmonary bypass $\left(30^{\circ} \mathrm{C}\right)$ and cold antegrade blood cardioplegic cardiac arrest. The left atrial tumor was initially inspected through the right atrium and the persistent foramen ovale. As described preoperatively, the tumor was polypoid, pediculated, and, atypically for a myxoma, located on the left lateral atrial wall, far away from the interatrial septum. An approach through the left atrial appendage was deemed to be better suited for complete resection. After complete excision with an adjacent strip of myocardium, the proximal left lower pulmonary vein and its orifice had to be reconstructed with a patch of fresh autologous pericardium. The patent foramen ovale was closed with a direct suture.

The patient had an uneventful postoperative course and was discharged on postoperative day 8 .

The tumor was $4.2 \mathrm{~cm}$ in diameter and consisted exclusively of epithelioid tumor cells in a myxoid matrix (Figure 1). The lesion was encapsulated in a fibrotic sheath. The tumor was resected in sano (R0). Immunohistochemically the tumor cells were negative for myxoma markers: CK 5/6, PAN-CK, actin, CD34, CD68, 